Abstract

\title{
Ensamble Sampling for Lattice Dynamics ${ }^{+}$
}

\author{
Paweł T. Jochym * and Jan Łażewski \\ Department of Computational Materials Science, Institute of Nuclear Physics PAS, ul. Radzikowskiego 152, \\ 31-342 Kraków, Poland \\ * Correspondence: pawel.jochym@ifj.edu.pl \\ + Presented at the 37th International Symposium on Dynamical Properties of Solids (DyProSo 2019), Ferrara, \\ Italy, 8-12 September 2019.
}

Published: 5 September 2019

Computational investigation of anharmonic and temperature-dependent aspects of lattice dynamics requires, among other things, replication of the conditions of thermal equilibrium. This requirement is very challenging when performing quantum mechanical calculations. Typically, it involves large number of atoms and long simulation times needed to approximate thermodynamical limit conditions. This is usually achieved by running a long molecular-dynamics calculation on the system, to thermalize all degrees of freedom, and selecting well-separated (independent) configurations from the obtained trajectory. While this approach provides good sampling of the configuration space of the system it is computationally very expensive and exceptionally wasteful. To obtain independent samples the selected times in the trajectory must be separated by multiple time steps-often tenths or hundreds. Thus, we are throwing away a large amount of computational time, often above $80 \%$, to obtain good sampling of the probability distribution in the configuration space. Furthermore, in the case of lattice-dynamical calculations, we are using only the positions from the trajectory-since we have usually no use for the velocity information. Together, this makes the described procedure limited to fairly small systems.

In this work we present an alternative scheme for creating a representation of the probability distribution in the configuration space, which aims to faithfully reproduce densities generated by the molecular dynamics, while being much more effective in terms of computational time. This approach uses well-known techniques of probability distribution modelling, and apply knowledge of the behaviour of the system in thermodynamic equilibrium to obtain low sample rejection rate in the procedure. This method, coupled with the effective-potential modelling of the interatomic forces provides a promising path to tackle problems of anharmonic and temperature-dependent lattice dynamics even in systems with large and complicated unit cells.

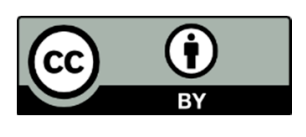

(C) 2019 by the authors. Licensee MDPI, Basel, Switzerland. This article is an open access article distributed under the terms and conditions of the Creative Commons Attribution (CC BY) license (http://creativecommons.org/licenses/by/4.0/). 